\title{
Control of integrated unit operations
}

\author{
José A. Chinea-Herranz, Manuel Rodríguez
}

\begin{abstract}
As a thermal separation method, distillation is one of the most important technologies in the chemical industry. Given its importance, it is no surprise that increasing efforts have been made in reducing its energy inefficiencies. A great deal of research is focused in the design and optimization of the Divided-Wall Column. Its applications are still reduced due to distrust of its controllability. Previous references studied the decentralized control of DWC but still few papers deal about Model Predictive Control. In this work we present a decentralized control of both a DWC column along with its equivalent MPC schema.
\end{abstract}

Keywords: Process Control, Thermally coupled distillation, Model Predictive Control.

\section{Main Text}

Basically, in every production process some of the chemicals go through at least one distillation column on their way from raw species to final product. Distillation is and will remain the main separation method in the chemical industry (there are more than 50,000 columns in operation around the world). Despite its flexibility and widespread use, distillation is very energy demanding. It can generate more than $50 \%$ of plant operating costs and it is the responsible of $3 \%$ of the energy usage in the U.S. (notice that the thermodynamic efficiency of a distillation column is between $5-20 \%$ ). In order to reduce this drawback new approaches and configurations have appeared.

The divided-wall column (DWC, the name is given because the middle part of the column is split into two sections by a wall) is an important example of process intensification and integration. DWC is very appealing to the chemical industry, with Montz and BASF as the leading companies. Kenig et al. state that there are more than 125 industrial applications nowadays and if the exponential trend continues there will be more than 350 by 2015 [1].

DWC can separate three or more components in one vessel using a single condenser and reboiler, hence reducing capital and operating costs compared to conventional twocolumn sequences. In fact, DWC can save up to $30 \%$ in the capital invested and up to $40 \%$ in the energy costs, particularly for close boiling species. DWC is considered to be on the path for energy conservation and green house gases emissions decrease.

DWC is not widespread due to distrust of its controllability. Its control is more difficult than the control of a conventional schema with two columns for the separation of ternary mixtures because there is more interaction among control loops. Besides, the absence of controllability could mean the absence of the energy savings if the optimal operation is not accomplished. The remaining of the paper is organized as follows. Section two discusses thermally coupled distillation columns, making emphasis in DWC columns. Section three presents the control strategies used in the paper. Section four applies decentralized (PID controllers) control and MPC to a ternary system separation. Finally, section five draws conclusions and introduces further work. 


\section{Energy integration in distillation}

Distillation systems have evolved from direct, indirect or distributed column sequences to thermally coupled systems and eventually to Petlyuk configurations [2] and DWC schemes (Fig. 1). Intermediate steps in this path include systems with heat pumps, prefractionators and side-stripers or side-rectifiers. The Petlyuk configuration and the DWC were aimed at the reduction of thermodynamic losses due to mixing streams, especially at the feed tray location.

DWC has more degrees of freedom (DOF) compared to a binary distillation column. This entails a complex design, but also presents extended optimization capabilities. If 3 product specifications are taken into account, DWC has 7 DOF's: distillate and bottoms flowrate, reflux ratio, reboiler duty, side-stream flowrate and vapor and liquid internal split ratios. 5 DOF's are used to stabilize 2 levels and 3 compositions while the remaining 2 DOF's are used for optimization purposes. Traditionally, liquid split ratio $\left(\alpha_{\mathrm{L}}=\mathrm{L}_{\mathrm{P}} / \mathrm{L}_{\mathrm{M}}\right)$ and vapor split ratio $\left(\alpha_{\mathrm{V}}=\mathrm{V}_{\mathrm{P}} / \mathrm{V}_{\mathrm{M}}\right)$ are optimization variables (Fig. $\left.1 \mathrm{c}\right)$. Vapor split ratio is usually fixed during the design stage because it is given by the pressure drop across both sides of the wall, which in turn depends on the stage type and geometry. The liquid split ratio is used as a control variable during operation by manipulating the flowrates leaving the bottom tray of the rectifying section.

The optimal design is given by the number of stages in the different sections of the DWC. The number of stages at both sides of the wall is usually the same but approaches with different number of plates have been reported. Olujic et al. presents a review on the different design approaches of DWC [3].

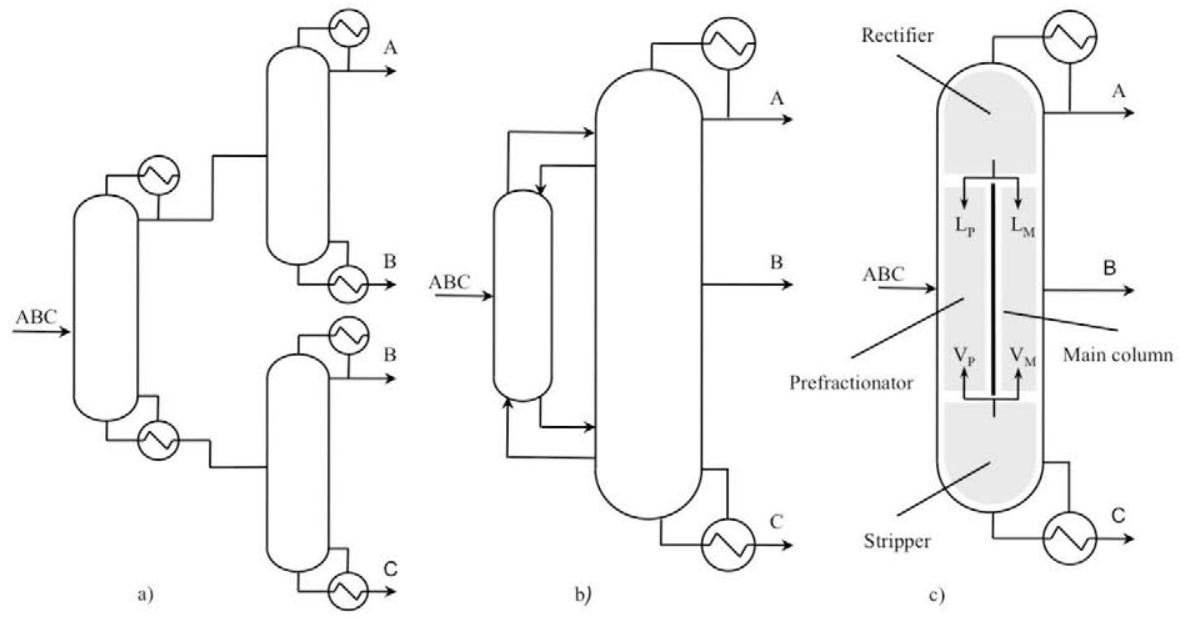

Figure 1. Evolution from a distributed sequence (a) to a Petlyuk configuration (b) and eventually to the DWC (c)

\section{Control strategies for Divided-Wall Columns}

Past and recent distrust on DWC controllability and flexibility is mainly due to the complex design of a control strategy. Maintaining product specifications while rejecting disturbances and loop interaction are the key concerns together with achieving significant energy savings. Otherwise, DWC advantages might disappear. 
Early references on DWC control were focused mainly on decentralized control. The first approaches extended PID control structures of traditional distillation columns by including liquid or even vapor split ratio among the manipulated variables. Wolff \& Skogestad [4] demonstrated that three-point control structures were feasible using PID controllers. Mutalib and Smith reported the first experimental application of decentralized control using temperature profile instead of composition measurements [5, 6]. Puigjaner et al. follow a research line in which multiple decentralized control studies are compared by using transfer functions obtained from shortcut modeling and dynamic matrix control is applied to DWC [7-9]. Kim [10] and Adrian [11] are among the first to use model predictive control but their approach is either experimental or shortcut modeling based. DWC has been also applied to complex distillation systems. Wang extended DWC to azeotropic distillation [12], while other references deal with extractive and reactive DWC $[13,14]$.

\section{Process studied}

The separation of n-pentane, n-hexane and n-heptane is carried out in a DWC. The rectifying, prefractionator and stripping sections have respectively 7,12 and 10 stages. The number of stages at both sides of the wall is the same. The feeding stage and sidestream withdraw are located at stage 12 in the prefractionator and main column respectively. The feed is assumed to be $180 \mathrm{kmol} / \mathrm{h}$ of a mixture $0.4 / 0.2 / 0.4(\mathrm{C} 5 / \mathrm{C} 6 / \mathrm{C} 7)$ in mole fraction at $300 \mathrm{~K}$ and product specifications are set at $98 \%$. The operation values are calculated to minimize the reboiler duty. The minimum energy consumption occurs at a liquid split ratio of 0.33 and a vapor split ratio of 0.625 . The resulting reflux and boilup ratios are 2.521 and 3.445 respectively. The steady state simulations are performed in Aspen Plus using the rigorous Radfrac model with a Chao-Seader property package. Sizing rules for reflux drum, bottoms and trays are taken from Luyben [15].

\subsection{Decentralized control}

The main advantage of decentralized control lies in its simple design and tuning. As less time and effort is needed on its development, it might be convenient for simple applications. However, PID ability to reduce interaction among control loops is reduced and as a result settling times and oscillation might compromise stable operation.

In decentralized control, the choice of variable pairings plays a fundamental role in the performance of the system. There are numerous references dealing with the best pairing. Variable pairing depends highly on the chemical system studied, the design and the product specification level. Nevertheless, the best result is traditionally associated to the strategy L/S/V-D/B, meaning that distillate, side-stream and bottoms specifications are controlled by reflux, side-stream and bottoms rate respectively while condenser drum and bottoms levels are maintained by distillate and bottoms rates respectively.

A special reference must be done on a paper by Luyben \& Lin applying a four composition PID control scheme [16], which is eventually extended to temperature profile control [17]. Luyben's control scheme minimizes indirectly energy consumption by maintaining heavy component concentration on top of the prefractionator at a minimum value. It is widely accepted that any minimal amount of heaviest component going out the top of the prefractionator causes an irreversible decrease on the side stream purity [4]. The same idea applies if the lightest component crosses the dividing wall at the bottom of the prefractionator, however the influence on product specification is not so important as this component will be present mainly in the vapor phase. As L/S/V-D/B gives the best performance according to most of the references and Luyben's control scheme accomplishes and indirect energy optimization, both 
approaches will be used. A PI controller is designed for each control loop, tuning parameters are shown in Table 1.

Table 1. Summary of tunning parameters for decentralized control

\begin{tabular}{llll}
\hline & Proportional gain & Integral time & Set point (mole frac.) \\
\hline Distillate composition control & 7.144 & 63.36 & 0.98 \\
Side-stream composition control & 121.37 & 38.28 & 0.98 \\
Bottoms composition control & 4.745 & 35.64 & 0.98 \\
Prefractionator composition control & 0.379 & 29.04 & 0.004 \\
\hline
\end{tabular}

4.2. Model Predictive Control (MPC)

There are few applications of MPC to integrated unit operation and especially to DWC. Some references highlight the enhanced performance of MPC while for others PID control gives better results. Relevant experience on the superiority of MPC is still limited. Theoretical and experimental comparisons between MPC and PID controllers should be done for a variety of chemical systems so as to reach a heuristic solution.

MPC present enhanced performance reducing oscillations, loop interactions and settling times. Besides the ability to include optimization constraints allows for the safety improvement. Nevertheless, MPC present several disadvantages: 1) it entails larger development cost and time, 2) it requires a deep knowledge of the process and 3) it requires the availability of a dynamic model representing the main features of the unit operation. The performance of MPC is directly proportional on the accuracy of the dynamic model and the adequate tuning of its parameters.

MPC control of the dynamic model for the DWC is carried out in Matlab with the MPC Toolbox. The step responses obtained from Aspen Dynamics are transformed to the Plant Model using the Systems Identification Toolbox. An ARX linear parametric model is found to represent the plant responses adequately

The MPC controller is tuned using the Tunning Advisor available in the MPC Toolbox. The Integrated Square Error function is used as the performance function for tuning.

\subsection{Performance comparison}

The decentralized control scheme has been tested to $\pm 10 \%$ disturbances in feed flowrate, temperature and composition as well as a simultaneous set point change in all product purities from 98 to $98.5 \%$ (mole frac.). Loop interaction is clearly demonstrated with the set point change experiment (Fig. 2 left). MPC performance is compared to decentralized control by carrying out the same simultaneous set point change.

The results are shown in Fig. 2 and clearly demonstrate the advantage of MPC in eliminating loop interaction and oscillations. Settling times for distillate and bottoms purity are significantly lower. For sidestream purity and prefractionator vapour composition the decentralized control scheme presents lower settling times.

\section{Conclusions}

In this paper the control of a DWC has been presented applying MPC as well as decentralized control. MPC superiority in eliminating loop coupling has been demonstrated comparing it to a decentralized control scheme. The same comparison has to be done with a decentralized control with decoupling strategies. In this case the development cost and time must be taken into account to compare both strategies 

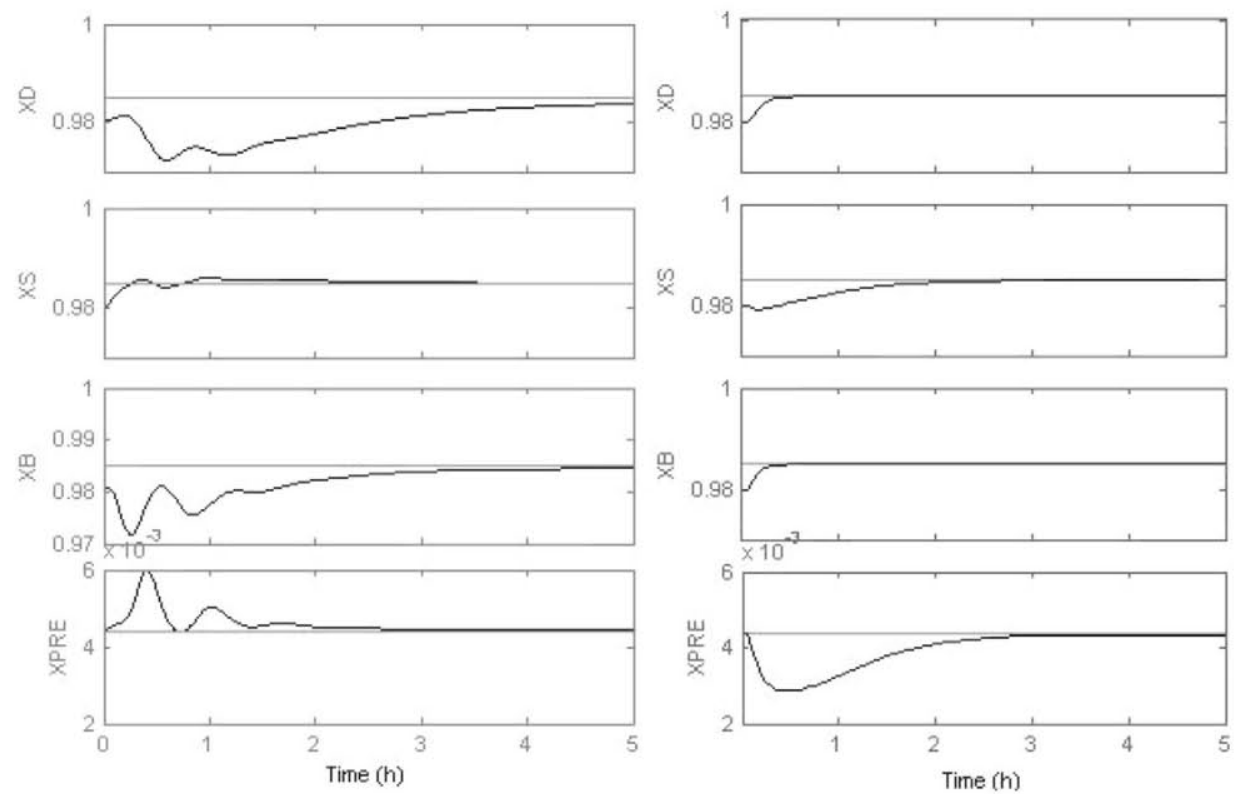

Figure 2. Set point increment of product purities from 98 to $98.5 \%$ for a decentralized control (left) and MPC scheme (right)

The industrial application of decentralized or model predictive schemes must be based on temperature profile control to avoid using composition analyzers, which in most of the cases are expensive and present slow responses.

The study of control strategies will be extended to complex schemes such as Kaibel columns, double-wall distillation columns (Sargent arrangements) or Agrawal columns.

\section{References}

[1] E. Y. Kenig, O. Yildirim, A. A. Kiss, Sep. Purif. Technol., 80 (2011) 403.

[2] F. B. Petlyuk, V. M. Platonov, V. M. Slavinskii, Int. Chem. Eng. 5 (1965) 555.

[3] Z. Olujic', I. Dejanović, L. Matijasević, Chem. Eng. Process., 49 (2010) 559.

[4] E. A. Wolff, S. Skogestad, Ind. Eng. Chem. Res., 34 (1995) 2094.

[5] M. Mutalib, M. Smith, Trans. IChemE. 76 Part A (1998) 308.

[6] M. Mutalib, M. Smith, Trans. IChemE. 76 Part A (1998) 319.

[7] L. Puigjaner, M. Serra, A. Espuña, Chem. Eng. Process., 38 (1999) 549.

[8] L. Puigjaner, M. Serra, A. Espuña, Ind. Eng. Chem. Res., 42 (2003) 1773.

[9] L. Puigjaner, M. Serra, A. Espuña, M. Perrier, Comput. Chem. Eng. 25 (2001) 859.

[10] Y. H. Kim, Chem Eng J., 85 (2002) 289.

[11] T. Adrian, H. Schoenmakers, M. Boll, Chem. Eng. Process., 43 (2004), 347.

[12] S. Wang, C. Lee, S. Jang, S. Shieh, J. Process Control, 18 (2008) 45.

[13] C. C. Bravo-Bravo, J. G. Segovia-Hernández, C. Gutiérrez-Antonio, A. L. Duran, A. BonillaPetriciolet, A. Briones-Ramírez, Ind. Eng. Chem. Res., 49 (2010) 3672.

[14] A. A. Kiss, H. Pragt, C. van Strien, Chem. Eng. Commun., 196 (2009) 1366.

[15] W. L. Luyben, Distillation design and control using Aspen ${ }^{\mathrm{TM}}$ simulation. Bethlehem, Pennsylvania. 2006.

[16] W. L. Luyben, H. Lin, Ind. Eng. Chem. Res., 48 (2009) 6034.

[17] W. L. Luyben, H. Lin, Ind. Eng. Chem. Res., 49 (2010) 189. 